\title{
Cartografia e patrimônio arquitetônico: a elaboração do atlas eletrônico do sítio histórico urbano da Lapa (PR)
}

\author{
Cartography and architectural heritage: the development of electronic \\ atlas of the historical city of Lapa (PR)
}

\author{
Naomi Anaue Burdal(*) \\ Marcello Martinelli
}

Resumo

O tema da pesquisa versa sobre a construção de um atlas eletrônico sobre o patrimônio cultural do sítio histórico da Lapa usando recursos de Cartografia e Geotecnologias. Sua problematização seria a falta de um conhecimento mais consistente do patrimônio arquitetônico do ponto de vista da Geografia e do Turismo da cidade da Lapa (PR). O objetivo principal desta pesquisa consiste em propor uma metodologia de atlas eletrônico para o patrimônio arquitetônico da Lapa (PR), associada à Geografia do Turismo e assistida pela cartografia temática. Os específicos são experimentar e compreender como as geotecnologias podem auxiliar na elaboração de uma metodologia para os atlas eletrônicos e a construção de um protótipo para o caso do patrimônio arquitetônico local; elaborar e experimentar cenários em 3D dos casarões do Centro Histórico e construir e experimentar roteiros e guias turísticos como opções de cenários que resultem em protótipos de guias de turismo e guias de patrimônio cultural.

Palavras-chave: atlas eletrônico; Lapa; patrimônio cultural.

\section{Abstract}

The theme of the research focuses on the construction of an electronic atlas on the cultural heritage of the historic site of Lapa using resources Cartography and Geotecnologies. His questioning was the lack of a more consistent architectural heritage from the standpoint of view to Geography and Tourism of the city of Lapa (PR). The main objective of this research is to propose a methodology for electronic

I MSc.; Geógrafa; Doutoranda em Geografia Humana pela Universidade de São Paulo, USP; Professora do Departamento de Geografia da Univerisidade do Centro-Oeste do Paraná, UNICENTRO, Guarapuava. Endereço: Rua Simeão Carvalho Varella de Sá,Vila Carli, CEP: 85040-080, Guarapuava, Paraná, Brasil. E-mail: naomi.burda@gmail.com. (*) Autora para correspondência.

2 Dr.; Geografia; Professor do Departamento de Geografia da Universidade de São Paulo, USP, São Paulo; Endereço: Av. Prof. Lineu Prestes, 338, Butantã, CEP: 01060-970, Caixa-postal: 2530, São Paulo, São Paulo, Brasil; E-mail:m_martinelli@superig.com.br 
atlas of the architectural heritage of Lapa (PR) associated with the Geography of Tourism and assisted by thematic cartography. Specifics are experiencing and understanding how geotechnology can assist in developing a methodology for the electronic atlas and construction of a prototype for the case of architectural heritage site and try and develop scenarios for the $3 \mathrm{D}$ houses the Historical Center and try and build road maps and tourist guides as options scenarios that result in prototypes tour guides and cultural heritage guides.

Key words: electronic atlas; Lapa; cultural heritrage.

\section{Fundamentação Teórico- Metodológica}

\section{Geotecnologias e o Tratamento de Dados para o Turismo e Patrimônio Cultural}

Um marco importante para o surgimento e a discussão sobre geotecnologias foi quando Roger Tomlinson inventou o termo "sistema de informação geográfica" para o Governo do Canadá no início dos anos 1960 (COPPOCK; RHIND, 1991). Com a evolução dessa área, houve uma discussão científica sobre a mesma, ganhando dimensão nas universidades, empresas, discussões na internet e entre os profissionais que trabalham com esse instrumento.

$O$ contexto que incentivou o desenvolvimento da geoinformação foi além das pesquisas acadêmicas, em consonância com uma mudança da própria sociedade da época que demandava por informações tecnológicas do espaço geográfico. Porém, de acordo com registros históricos havia uma necessidade anterior maior de raciocínio dos sistemas espaciais, discutidos por Ptolomeu e Kant muito antes do desenvolvimento das geotecnologias (FORESMAN, 1998).

Para Abadala (2009, p. 104) a passagem da cartografia tradicional para a cartografia digital ocorreu quando houve mudanças no uso das técnicas e materiais no tratamento cartográfico. Primeiramente, a forma tradicional usava a caneta, tinta e o papel, para então aplicar o uso intensivo de computadores para a criação de mapas. $\mathrm{Na}$ cartografia tradicional eram exigidas aptidões físicas humanas, tais como habilidade manual, acuidade visual, entendimento espacial. Para Martinelli (2011), a evolução da cartografia analógica para digital não alavancou apenas na produção de mapas, mas auxiliou na tomada de iniciativas para novas metodologias de representações a partir de dados georreferenciados.

$\mathrm{O}$ início da era digital cartográfica ocorre no pós Segunda Guerra, com o surgimento de programas de computador Computer Aided Cartography (CAC) e a fabricação de equipamentos específicos para a cartografia (mesas digitalizadoras, plotters coloridos e scanners de grande formato). Há também o desenvolvimento de conhecimentos voltados para a tecnologia computacional empregando a cartografia (restituição analítica, tratamento digital de imagens, por exemplo).

Esses elementos contribuíram para a formação da Cartografia Digital e da tecnologia dos SIG's, e a produção e estudo de informações especializadas (GOODCHILD，1990; TAYLOR, 1994). Uma dessas especialidades é a acessibilidade que a Cartografia oferece através da Cartografia Tátil e programas 
como o Mapavox ${ }^{3}$ (BORGES; FREITAS; VENTURINI, 2011, p. 110).

Com o rápido crescimento das geotecnologias e também dos SIG's houve o ressurgimento de discussões de cunho epistemológico da Geografia. Alguns autores discutem se essa área é uma ciência ou ferramenta, tais como Wright, Goodchild e Proctor (1997) que argumentam que precisamos distinguir entre SIG como uma ferramenta para ser ensinado em nível de graduação, ou uma ciência e, portanto, uma especialidade de pesquisa legítima de professores e alunos de pós-graduação.

Há autores que defendem a grande contribuição que o computador influenciou na geografia como disciplina acadêmica, onde o computador contribui muito mais que a parte instrumental e sim para a formação de uma nova metodologia geográfica para a pesquisa do espaço (TOMLINSON, apud VEREGIN, 1995, p. 103).

Quando começaram a relacionar as geotecnologias com a ciência geográfica, já que ambas tratam de um objeto de estudo em comum, a análise estatística do espaço geográfico, emergiu uma necessidade de uma discussão teórica aprofundada sobre tal tema. A ciência da informação geográfica ascende e aproxima ciências como a fotogrametria, cartografia, geodésia e análise espacial.

Para Pina e Santos (2000) os Sistemas de Informações Geográficas consistem em "sistemas computacionais, usados para o entendimento dos fatos e fenômenos que ocorrem no espaço geográfico”. O diferencial deste sistema para outros é que há uma capacidade de formar grandes conjuntos de

3 Um conjunto de microchaves e mapas didáticos que disponibilizam dados sonoros em materiais táteis ou não. dados espaciais, organizados através de um bando de dados, que podem ser integrados ou não.

Desde o seu surgimento até a atualidade, existe um déficit de abordagens teórico-metodológicas a respeito do SIG. Tomado somente como instrumento, técnica e produtos, ficam restritas as reflexões sobre o que é tal sistema, suas funções e aplicações dentro das diversas áreas.

Temos uma emergência de discussões nessa linha, pois um usuário que apenas sabe usar tal instrumento e não sabe interpretálo torna a sua pesquisa vazia. $\mathrm{Na}$ Geografia há uma dificuldade em inserir o SIG como assunto teórico e metodológico existindo poucas publicações que insiram críticas epistemológicas, políticas e éticas (MATIAS, 2010, p.90).

Nesse estudo há também a necessidade de acesso democrático de tal geoinformação, que está sendo superada pela divulgação dos dados via internet (eletrônico). Mas questiona-se também a confiabilidade de tais dados disponibilizados na rede, pois as listas de discussão e redes de colaboração em SIG são feitas tanto por geografia e profissionais de áreas afins, quando por pessoas que se interessam por tal assunto mas não possuem formação específica.

Alguns períodos que marcaram o desenvolvimento do Sistema de Informação Geográfica foram de acordo com Câmara et al. (1996), foram os seguintes: A primeira fase estava baseada em um CAD cartográfico, baseado na Cartografia Tradicional, contendo um banco de dados cujo suporte é o mapa, os chamados Sistemas Orientados e Projetos. A segunda fase foi baseada em banco de dados geográficos para o uso de clientesservidor, ligados a bancos de dados maiores e ao processamento digital de imagens. Já 
a terceira fase teve o suporte das bibliotecas digitais geográficas e os centros de dados geográficos, que permitiam a elaboração de bancos de dados geográficos extensos.

Entre as diferentes concepções a respeito do SIG podemos citar a de Zeiler (1999) que aplica uma visão ampla do assunto:

Um Sistema de Informação Geográfica é a combinação de pessoas qualificadas, dados espaciais e descritivos, métodos analíticos, software e hardware computacionais - tudo organizado para automatizar, gerenciar e prover informação para apresentação geográfica.

Rego (2007, p. 84) destaca o SIG através de sua importância para o manuseio de dados espaciais:

[...]um SIG cria uma realidade virtual do espaço geográfico composta por camadas de informações em que cada um representa um aspecto desse espaço geográfico. [...] essas camadas se cruzam espacialmente na medida em que todos esses dados são georreferenciados, ou seja, dentro de um mesmo sistema de referência geográfica.

Tal relevância está na interdisciplinaridade de um SIG. Podem ser feitos estudos pautados nas áreas do planejamento territorial e planejamento turístico, quando são úteis os mapas estatísticos.

Burrough (1996, p. 6) tem uma ampla argumentação, pois para ele o SIG é "um conjunto poderoso de dispositivo para coletar, armazenar, recuperar à vontade, transformar e exibir dados espaciais do mundo real atendendo um conjunto particular de propósitos". Nessa definição há uma integração tanto da parte instrumental quanto da representação do mundo real com objetivos específicos. Ainda acrescenta que há um desenvolvimento técnico e conceitual do SIG, que une dados da Cartografia; computadores assistidos por desenhos e gráficos de informática; levantamento e fotogrametria; análises espaciais usando dados rasterizados para mapas temáticos e; tecnologia de sensoriamento remoto.

Tomlin (1990,p.XI) “uma configuração de hardware e software computacional especificamente projetado para a aquisição, manutenção e uso de dados cartográficos". Nesse caso procurou-se potencializar apenas a parte técnica, incluindo a soma de hardware e software específicos.

Carter (1994) classifica como "uma entidade institucional, refletindo uma estrutura organizacional que integra tecnologia com banco de dados, especialistas e suporte financeiro continuado".

Berry (1987) define o SIG como um "sistema automatizado de informação espacial, interiormente referenciado, projetado para administração de dados, mapeamento e análise" Aqui o autor cita o diferencial de um sistema SIG para um sistema CAD que permite a espacialização dos dados.

Smith et al. (1987) conceitua o SIG como um sistema de base de dados cuja função é indexá-los espacialmente, no qual um conjunto de processos é criado a partir de perguntas que são feitas sobre as entidades espaciais na base de dados.

Dentre as definições de SIG, algumas são idênticas, outras se limitam em apenas como um sistema de informação. Seguem quatro linhas de conceituação desses produtos:

- Uma reducionista, que considera apenas a contribuição dos equipamentos para o sistema, não citando a contribuição de uma equipe profissional no processo, como a de Tomlin. 
- A que trata a informação geográfica como uma estrutura organizacional incluindo especialistas, tal como a definição de Carter.

- Destaque para a presença de dados georreferenciados como a de Berry.

- Um software que tem como função principal a anexação de informações espaciais e suas análises como Smith et al.

Câmara et al. (1996) montou um esquema para compreensão das interfaces do Sistema de Informação Geográfica para mostrar a integração entre os elementos da interface (softwares), entrada e integração dos dados (via GPS, teclado ou digitalização em tela), consulta e análise espacial (através de ferramentas dentro do SIG, tais como a query), visualização e plotagem (através da tela do computador e impressão) e a gerência dos dados (através de um banco de dados integrado).

A difusão das geotecnologias está se ampliando cada vez mais. O que antes era domínio técnico das geociências e das ciências cartográficas, instituições públicas ou empresas de aerofotogrametria passa a ser de atividades cotidianas de usuários comuns (MATIAS, 2010, p. 82). Isso vem se comprovando com a disponibilização de imagens de satélite e mapeamento em redes de internet, as extensões que o Google vem disponibilizando (Google Maps, Google Sketchup. Google Earth), as redes colaborativas que constroem sigs e disponibilizam extensões e tutoriais para usuários menos preparados cientificamente.

Esse fato ocorre, pois há uma "necessidade mais ampla na sociedade contemporânea da produção de informações de forma rápida e precisa sobre o espaço geográfico" (Ibid., p. 82). Sem ter um conhecimento do mesmo, fica difícil seguir um planejamento ou uma ação consequente que atenda aos objetivos dos diversos grupos sociais envolvidos.

O papel das geotecnologias para a criação de trabalhos de mapeamento e estudos do espaço geográfico possui alguns elementos:

1. Conhecimentos e técnicas que permitam tratar em um mesmo ambiente computacional dados espaciais e não espaciais;

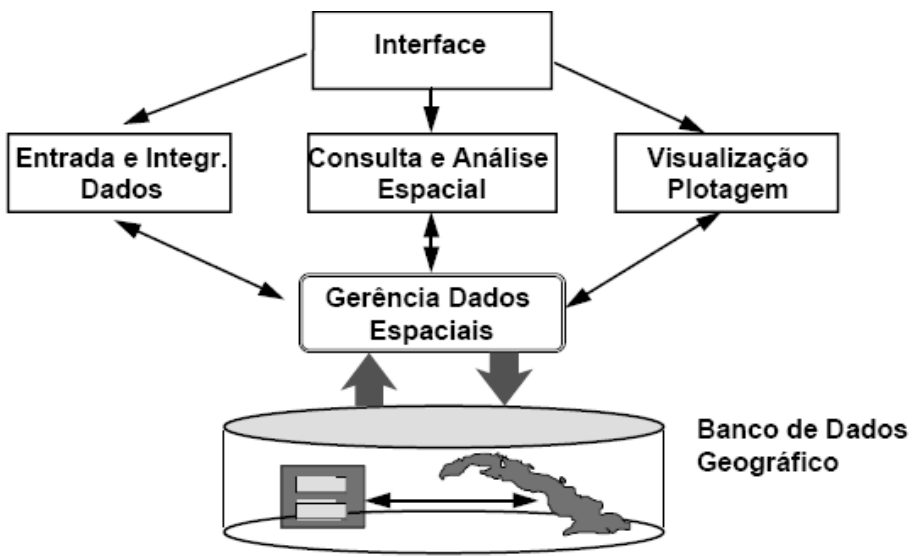

Figura 3.1 - Estrutura Geral de Sistemas de Informação Geográfica

Figura I. Estrutura Geral de Sistemas de Informação Geográfica Fonte: CÂMARA et al., 1996 
2. Aquisição de dados de diversas fontes, diferentes extensões e padrões;

3. Produção de informações georreferenciadas de forma ágil e precisa.

A especialização dos lugares leva a uma divisão internacional do trabalho e uma produção e circulação de informações. As informações segundo Santos e Silveira (2008, p. 95) encontram-se,

globalmente organizada, que se constrói e se difunde com instrumentos de trabalho específicos, isto é, sistemas técnicos sofisticados, exigentes de informação e produtores de informação sobre $o$ território.

O mundo atual constituiu o chamado tempo real. A informatização permitiu um novo arranjo da organização do trabalho, as redes se especializaram e a informação circula por ela através dos fluxos.

Mesmo com esses avanços na informação, é necessário relacionar a cultura do lugar com a cultura produzida no lugar. Esta permite uma contiguidade territorial e a dinamização da economia local. Esse conhecimento cultural cria "uma base de vida que amplie a coesão da sociedade civil, a serviço de um interesse coletivo" (SANTOS, 2008, p. 228).

Loureiro (1991) reforça que o momento histórico que estamos vivendo tem influência de Aque "as transformações são determinadas pelo progresso da informática e da microeletrônica, substituindo o homem no trabalho direto e tornando as atividades produtoras de informação e conhecimento, a mais importante economicamente e socialmente falando".

Martin (1995), se dirige à atualidade como um paradigma sócio-econômico, que tem como elemento principal a informação, pois estão formados pela informação, comunicação, microeletrônica, computadorização, produtos feitos com base em conhecimento e padrões de consumo.

Harvey (1992) em suas abordagens inseriu a importância do uso da tecnologia da informação e o mapeamento digital para representação do espaço. Este reforça a análise do instrumental cartográfico para o entendimento de estratégias e controle das dimensões temporal e espacial. Nesse contexto, surge em 1970 o aparato da infraestrutura geotecnológica como a série de satélites LANDSAT e o sistema NAVSTAR/GPS.

A evolução das tecnologias no ramo da cartografia reafirma o que foi defendida por Taylor (1987, p. 359) "a disciplina da cartografia está em estado de mudança rápida, dirigido principalmente pela incrível velocidade de desenvolvimento tecnológico associado com a revolução da informação".

As discussões teóricas que existem acerca das Geotecnologias estão relacionadas ao termo Geoprocessamento. Rodrigues (1987) a definiu como "conjunto de tecnologias de coleta e tratamento de informações espaciais e de desenvolvimento, e uso, de sistemas que as utilizam". Esse conceito aproxima-se dos conceitos de SIG discutidos anteriormente, o que demonstra que há uma certa indefinição das diferenças entre um e outro.

Teixeira, Moretti e Christofoletti (1992) conceituam o geoprocessamento como um "ambiente tecnológico [...] cuja área de atuação envolve a coleta e tratamento da informação espacial, assim como o desenvolvimento de novos sistemas e aplicações”. Faz um tratamento específico do desenvolvimento tecnológico do termo, sem informação teórica. 
Câmara e Monteiro (1999) usam termo geoprocessamento no sentido de "uma disciplina do conhecimento que utiliza técnicas matemáticas e computacionais para o tratamento de informações geográficas." Aqui é inserida a questão disciplinar, onde o geoprocessamento pode contribuir no ensino e no entendimento das representações geográficas no ambiente computacional.

Rocha (2000) indica o

Geoprocessamento como transdisciplinar, pois envolve vários ramos do conhecimento tanto na sua construção (informática, eletrônica) quanto na análise dos dados (cartografia, geografia, geologia, biologia, entre outros).

[...]uma tecnologia transdisciplinar, que, através da axiomática da localização e do processamento de dados geográficos, integra várias disciplinas, equipamentos, programas, processos, entidades, dados, metodologias e pessoas para coleta, tratamento, análise e apresentação de informações associadas a mapas digitais georreferenciados. (ROCHA, 2000, s.p.).

Os produtos das Geotecnologias estão divididos em mapas digitais, maquetes virtuais, modelos digitais de terreno, atlas digitais, atlas eletrônicos e outros.

A fonte de dados principal do geoprocessamento são os elementos, fatos e espaço geográfico. Sendo o espaço geográfico objeto de estudo da Geografia, cabem perfeitamente estudos através das Geotecnologias.

A criação de produtos do Geoprocessamento permite a abstração da realidade para o papel/tela, o que permite o conhecimento de lugares distantes, espaços de difícil acesso. Sua facilidade de manipulação e atualização contribui para estudos geográficos. É um instrumento de fácil acesso, porém exige treinamento técnico e embasamento teórico.

Com base em Matias (2004), o advento das Geotecnologias (incluindo a Cartografia Digital, Sensoriamento Remoto, Sistema de Posicionamento Global - GPS, Sistemas de Informações Geográficas - SIG’s) existiu em um contexto cuja informação necessitava de uma infraestrutura que auxiliasse na aquisição, processamento e análise de informações sobre o espaço geográfico que resultasse na produção e tomada de decisão por parte de quem usaria tal informação. Para esse autor,

as geotecnologias fazem parte do conjunto de objetos e de ações necessárias à [re]produção do espaço geográfico na atualidade, daí sua utilização e análise constitui elementos primordiais para compreensão desse processo (MATIAS, 2004, s.p.).

Para Fitz (2008,p.11), as geotecnologias são compreendidas como "novas tecnologias ligadas às geociências e correlatas, as quais trazem avanços significativos no desenvolvimento de pesquisas, em ações de planejamento, em processos de gestão, manejo e em tantos outros aspectos relacionados à estrutura do espaço geográfico". Trata-se de uma definição atualizada, visto que vários profissionais podem trabalhar com questões espaciais, porém devem estar preparados tanto teórica quanto metodologicamente para essa tarefa.

De acordo com Buzai (1999, 2000, apud FITZ, s. d., p.) ainda há uma resistência nos eventos dos profissionais em Geografia quanto a discussões das denominadas Geotecnologias, ou as "novas tecnologias ligadas às geociências e outras correlatas". E sua concepção de Geotecnologias está elucidada a seguir: 
La Geotecnologia presenta una nueva forma de ver el mundo. Una nueva forma de ver la realidade que la Geografía le provee al resto de las disciplinas. Bajo estas consideraciones el Paradigma Geotecnológico tiene existencia, pero no como paradigma de la Geografía, sino como paradigma Geográfico de alcance interdisciplinario y al servicio total del hombre (BUZAI, 2004, p. 188).

Em suma, entender sobre geotecnologias implica em não apenas dominar a parte instrumental (software, hardware, equipamentos), mas também leva a entender "o porquê, o como, o para quê e o para quem do advento tecnológico". Os motivos que levaram à iniciação e ao desenvolvimento dessa tecnologia, o momento histórico e quais as reais necessidades para tal. O mais importante aqui é apreender as geotenologias como auxiliares na representação do espaço, das práticas sociais e dos espaços de representação do capitalismo no espaço geográfico (MATIAS, 2004, s. p.).

\section{Mapeamento e Atlas: uma proposta para a Cartografia do Turismo e Patrimônio Cultural}

Para entendermos a representação do espaço do patrimônio cultural para o turismo temos que ter em mente duas análises, uma que ocorre através dos mapas e outra, complementar, através dos atlas.

Respondendo a uma questão metodológica sobre tal tema, a primeira fase para conhecimento do patrimônio arquitetônico de uma cidade ocorre através da busca dos registros de bens culturais que têm destaque patrimonial. O levantamento de materiais cartográficos através de plantas e cartas que estão disponíveis, para embasar a continuidade do trabalho.
A segunda etapa consistirá na delimitação da área de trabalho, considerando o contexto espacial no qual está localizado. Portanto os elementos arquitetônicos não são o suficiente para essa decisão, também devendo incluir "características físico-territoriais, por exemplo, topográficas, pedológicas [...] que características socioeconômicas que definem o perfil da população que vive na localidade (renda, faixa etária, escolaridade etc.)" (MATIAS, 2010, p. 96). Agregam-se aos dados características históricas do local, tais como características arquitetônicas, dados cadastrais, legislação patrimonial e documentos iconográficos.

A vantagem em se construir tais dados através das Geotecnologias é que poderão ser feito primeiramente o mapeamento e posteriormente a análise do patrimônio cultural arquitetônico. A disponibilização de dados georreferenciados.

Em termos de geotecnologias de mapeamento, pode-se apontar a criação de atlas digitais, que utilizam e unem em um só documento vários documentos atuais como fotografias aéreas, imagens de satélite, bancos de dados e registros fotográficos; ortofotos digitais, ortofotocartas, mapas digitais, imagens de satélite, bancos de dados e registros fotográficos; sendo este instrumento essencial como auxílio à gestão do patrimônio cultural (BURDA, 2009, p. 100).

Quanto ao uso de tecnologias de mapeamento, podemos citar a aplicação de medições através de receptores de Sistema de Posicionamento Global (GPS), pois definem a localização de monumentos arquitetônicos e edificações tombadas com precisão centimétrica. No caso de geração de volumetria do terreno, temos a utilização de teodolitos e estações totais com perfilhamento 
a laser, que permite uma "varredura" do terreno em posição planialtimétrica $(\mathrm{X}, \mathrm{Y}, \mathrm{Z})$.

Para um mapeamento generalizado, as imagens de sensores orbitais de satélites como Ikonos, Quickbird, Eros, Orbview servem para interpretação das formas de ocupação e uso da terra existente no lugar estudado (MATIAS, 2010, op. cit.).

A figura mostra uma imagem do Centro Histórico da Lapa que, utilizando uma imagem do programa Google Earth que continha nas informações do IPHAN, foi georreferenciada e gerado um mapa do estado de conservação pelo programa ArcGIS (ESRI, 2012).Também é mostrado parte do banco de dados que foi criado para associar as edificações com seus elementos complementares (rua, conservação, fotos e outros).

Das características topográficas da área do centro histórico da Lapa foram geradas formas topográficas de seu centro histórico, onde podem ser estudadas as feições físico-territoriais do sítio onde se encontra o patrimônio e as formas de subsídio para a capacidade do meio físico (áreas a serem tombadas, degradação do patrimônio por questões geoambientais) (MATIAS, 2010).

Através da rotação dos eixos $\mathrm{X}, \mathrm{Y}$ e $\mathrm{Z}$ dos modelos digitais de terreno, adicionando uma variável altimétrica, pode-se simular uma posição com visão vertical (Figura 5), uma visão oblíqua com visada leste (Figura 6), para que o observador consiga verificar os diversos pontos de vista da realidade.

Com o mapeamento das formas de uso e ocupação da terra na cidade pode-se realizar estudos sobre: produção de um inventário sobre o patrimônio cultural construído, diagnóstico sobre o uso da terra na área tombada, classificação de edifícios contra

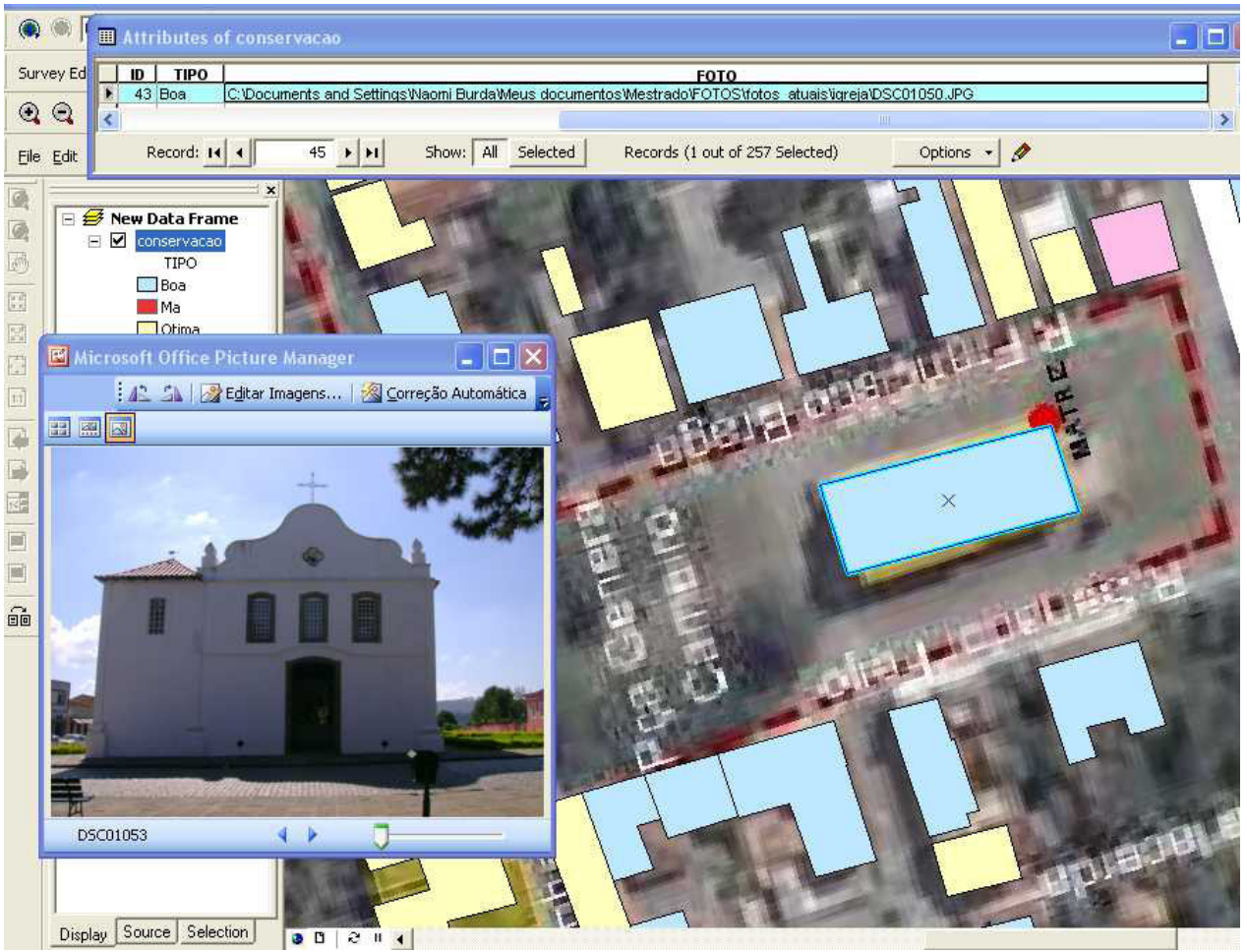

Figura 2. Estado de conservação das edificações do centro histórico Fonte: BURDA, 2009 


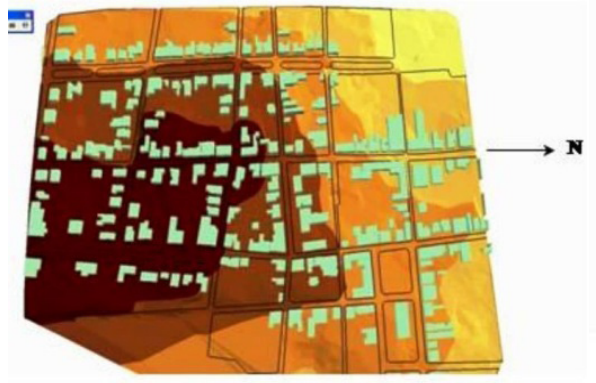

Figura 3. Visada vertical Fonte: BURDA (2012)

incêndios, estudos de impactos causados no patrimônio cultural com a construção e a modificação de edifícios, proteção e monitoramento do patrimônio cultural, gestão informatizada do patrimônio, divulgação através de multimídia, definição de roteiros histórico-culturais,.valorização de áreas de valor cênico (BURDA, 2009, op. cit.).

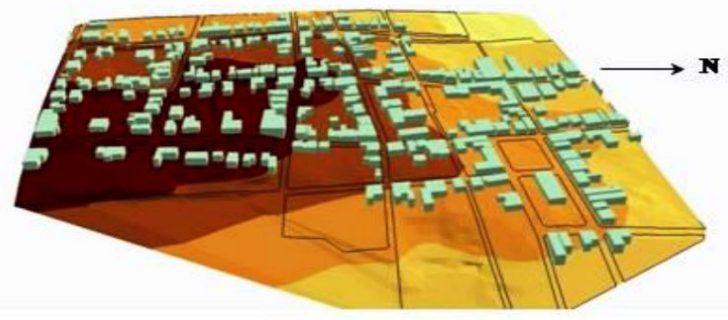

Figura 4. Visada oblíqua de Leste para Oeste

A proposta de montar roteiros turísticos contendo os principais atrativos da cidade pode indicar para o turista os itinerários mais rápidos e a indicação sobre como chegar a esses espaços torna a representação pela Cartografia Turística mais explicativa. Algumas características que podem ser trabalhadas são os roteiros arquitetônicos

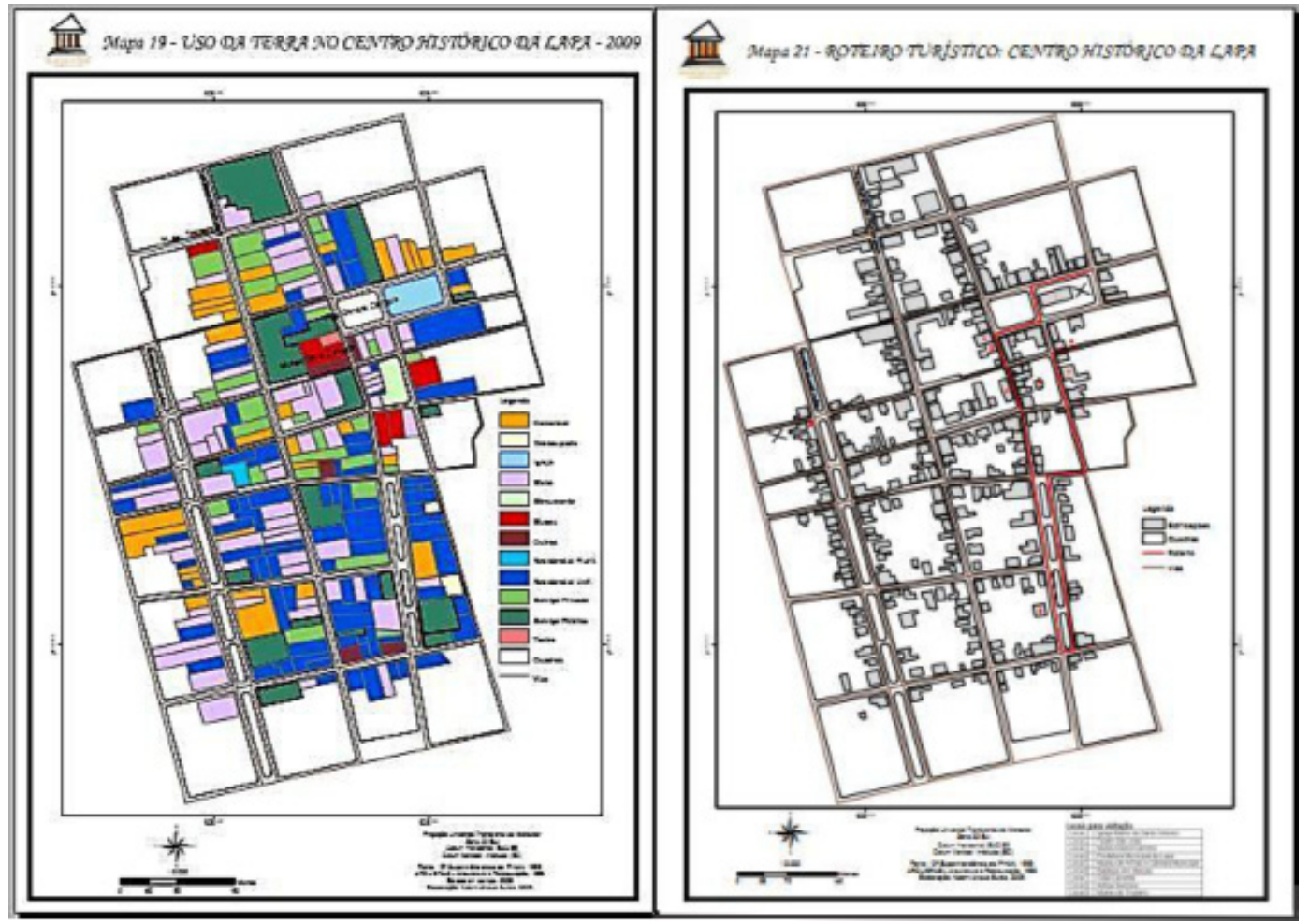

Mapa I. Ocupação e uso da terra na Lapa (PR) - 2009

Mapa 2. Sugestão de roteiro turístico na Lapa (PR) Fonte: BURDA, 2009 
socioculturais, arquitetônicos religiosos, dos principais serviços e gastronômico.

Mediante as inúmeras possibilidades e recursos das Geotecnologias para burilar os dados para auxiliar nas atividades do turismo, reforça-se nesse momento a importância deste trabalho. O aporte para a Geografia do Turismo identificada através de atlas eletrônicos no Brasil ainda é uma perspectiva inovadora, e pede-se que haja mais atenção a estudos dessa vertente.

Os atlas surgiram da necessidade dos homens possuírem informações em conjunto sobre o seu território, mediante uma coletânea prática de mapas. Através da coleção de plantas, cartas e mapas houve uma verdadeira difusão do conhecimento cartográfico na história mundial. Primeiramente, os gregos reuniam coletâneas de mapas em forma de atlas universais. Claudio Ptolomeu já organizava na época mapas com um "banco de dados" contendo lista das localidades do mundo (MARTINELLI, 2011).

Os atlas tiveram o papel de base para gestão e estratégia do território para os reis, governadores e pessoas que detinham o poder; pois estes visavam expandir o seu domínio através do conhecimento do território que pretendiam controlar.

Quanto ao desenvolvimento dos atlas, temos na Antiguidade Clássica a presença de um atlas que pode ser comprovado na obra de Cláudio Ptolomeu cujo Tratado de Geografia, incluiu uma coleção de mapas contendo um mapa-múndi e mais vinte e seis mapas temáticos do mundo antigo. Essa imagem de centralidade da Europa é referência na confecção de outros atlas e livros escolares de Geografia (MARTINELLI, 1999, p. 7-9).

Os atlas de referência surgiram em propostas como a do Atlas de Blaeu (Bleaw) de 1635 estruturado em 12 volumes, com mapas temáticos complementados por textos geográficos e históricos (Ibid., p. 10).

A partir do século XIV surgiram os Atlas Nacionais e Regionais, contendo mapas temáticos com objetivos seletivos. Eram símbolos de Estado e aqui se destaca $\mathrm{o}$ atlas inglês "Atlas of England and Wales" de Saxton (1579) e o Atlas "Theatre Francoys" de Bouguereau (1594), ambos representando os territórios da Inglaterra e França e afirmando seu poder e domínio territorial (Ibid, p. 12). Mais um impulso dado aos atlas foi aquele efetuado a partir da I Guerra Mundial, quando os Estados necessitavam de mapas para registrar suas mudanças e conquistas territoriais e uma efetiva aplicação da cartografia temática (Ibid., p. 13). Na Europa Ocidental esses atlas serviriam para afirmação da soberania e a partir de 1940, difundiram acentuadamente.

De acordo com Delazari (2004), um atlas eletrônico deve contar com a interatividade e atingir um grande número de pessoas. Isso pode ocorrer através de mídias digitais, do ambiente da Internet e outros. A mesma autora baseada em Kraak e Ormeling (1998, apud DELAZARI, 2004) afirma que existem 3 tipos de Atlas eletrônicos:

- Atlas eletrônicos "só para ver" (viewonly): são formas eletrônicas do atlas analógico, porém permite o acesso de forma aleatória. Estes têm menor custo e maior divulgação, pois são em cd-rom;

- Atlas eletrônicos interativos: o usuário tem acesso a manipulação de dados, podendo no ambiente interativo modificar cores, $o$ número de classes e outros. Não mudam o trabalho original;

- Atlas eletrônicos analíticos: levam a uma exploração mais aprofundada do ambiente digital. Os dados podem ser integrados e os usuários não se limitam apenas aos 
temas do atlas. As operações de SIG, como áreas, buffers, confecção de novos mapas são possíveis.

O atlas eletrônico irá representar não somente a transição do nível digital para o virtual, mas o emprego de questões metodológicas que irão se basear na Cartografia. Esta deve resolver os processos que estarão em dinâmica no patrimônio cultural da Lapa.

A elaboração de um atlas eletrônico começa na definição de seu conteúdo coerente com a proposta contida no objetivo geral do projeto da obra, na escolha dos dados que irão compor os temas de seus mapas. O registro dos bens patrimoniais, a organização arquitetônica e a localização destes por meio de cartas e mapas é o primeiro passo. Posteriormente, a seleção de imagens de sensores orbitais com resolução espacial adequada. A visualização do sítio urbano onde se localiza o patrimônio arquitetônico através de representações digitais tridimensionais contribui para uma interatividade no ambiente virtual; onde o usuário, por exemplo, pode realizar um "passeio virtual" no sítio histórico também é relevante (MATIAS, 2010).

Será necessário buscar um servidor de mapas via web, para inserção dos dados geoespaciais, divulgação e atualização dos mesmos. Alguns exemplos já foram empregados através dos serviços de Web Mapping Service e Web Feature Service.

A definição de novos caminhos na construção do Atlas Eletrônico será elaborada durante o preparo da tese, mantendo a originalidade do projeto e a busca de novas rotas metodológicas a respeito de atlas eletrônicos.

A vantagem para o turista de pesquisar um local em um atlas eletrônico está na facilidade de acesso a um material prévio ao seu local de visitação.Pode-se pensar que este é um fator de repulsão do turista, pois vendo o local de visitação em casa o mesmo se desestimulará a realizar tal visita. Porém, tendo acesso a tais informações, esse instrumento contribuir como fator de atração ao local, pois tendo um conhecimento dos atrativos e elementos do patrimônio cultural, por exemplo, o mesmo terá mais estímulo a procurá-lo.

Com o acesso à internet fica facilitado a divulgação de mapas turísticos e também destacar aspectos da cartografia, turismo e patrimônio cultural.

\section{Materiais e Métodos}

Os materiais utilizados para a pesquisa são o software de Geotecnologias licenciado ArcGIS Desktop da Esri, software Google Sketchup, internet e modelos de páginas virtuais.

Os métodos resumem-se em levantamento e revisão bibliográfica da literatura relevante,

sobre geografia, turismo, patrimônio cultural, cartografia, atlas eletrônicos, multimídias, geotecnologias e outros; para construção da argumentação teórica na pesquisa; pesquisa e tratamento de dados de atributos, de dados numéricos, imagens de satélites e/ou fotografias aéreas, para usar como base para o mapeamento; pesquisa e experimentação de programas e sites para o auxílio na construção do atlas eletrônico; atualização da cartografia já existente e complementação do mapeamento dos temas que constarão no atlas eletrônico; trabalho de campo com registro fotográfico (máquina digital) e montagem do catálogo (disponível digitalmente), para balizar os cenários 3D; elaboração dos cenários $3 \mathrm{D}$, contendo as alturas e volumetrias dos casarões; produção de um protótipo do atlas eletrônico; avaliação da possível divulgação do atlas eletrônico; e produção da tese. 


\section{Resultados e Discussão}

Dentro os resultados prévios que podem ser citados, menciona-se aqui os modelos de volumetria disponíveis no programa Google Sketchup. Sua construção ocorreu a partir de medições em campo da altura dos casarões tombados, registro em banco de dados do programa ArcGIS Desktop e importação para o programa Google Sketchup. O modelo volumétrico está disponível na figura abaixo, aqui dando uma maior ênfase aos casarões, pois anteriormente já foram demonstradas as visadas oblíquas.

Outro resultado obtido foi a montagem de um atlas protótipo que foi submetido à banca de qualificação para discussão e melhorias. Ressalta-se aqui que o trabalho está na fase de estruturação do atlas eletrônico e este modelo não é o modelo final, apenas uma sugestão do que poderia ser o site do atlas eletrônico.

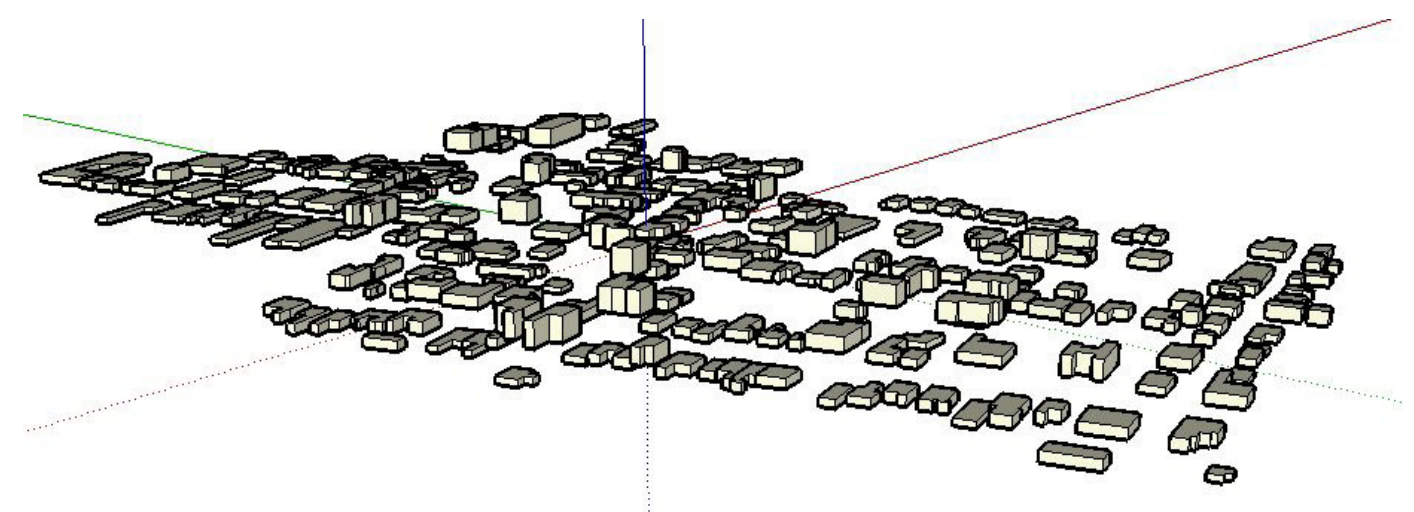

Figura 5. Exemplo de modelos no programa Google Sketchup Fonte: BURDA, 2011.

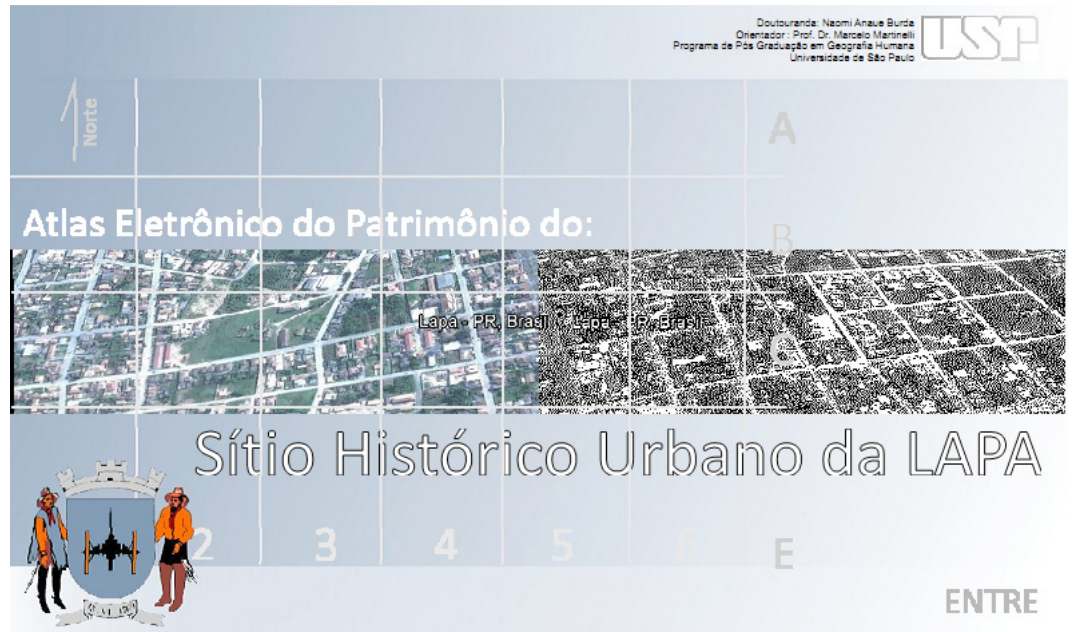

Figura 6. Página inicial do atlas protótipo

Fonte: BURDA, 2012 


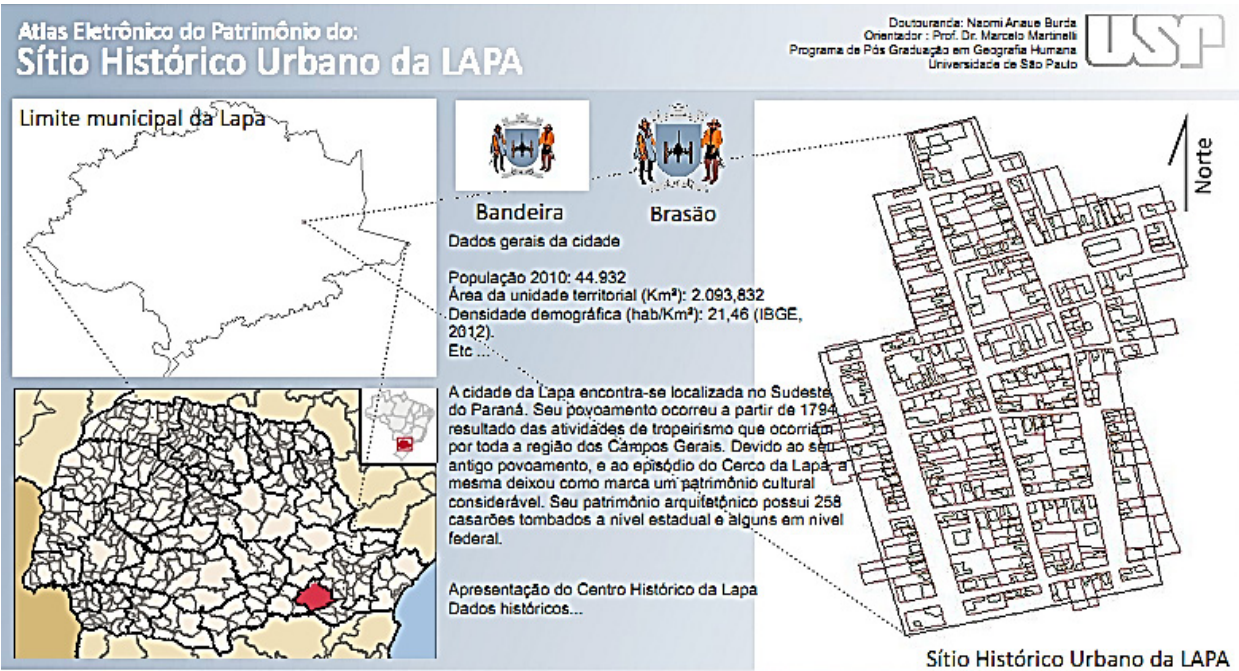

Capa | Apresentação | Mapa índice | Localização | Mapas Interativos | Roteiros Interativos | Videos

HOME

You ereated this PDF from an application that is not licensed to print to novaPDF printer (http:/fiwww.novapdt.com)

Figura 7. Localização da área de estudo do atlas protótipo

Fonte: BURDA, 2012

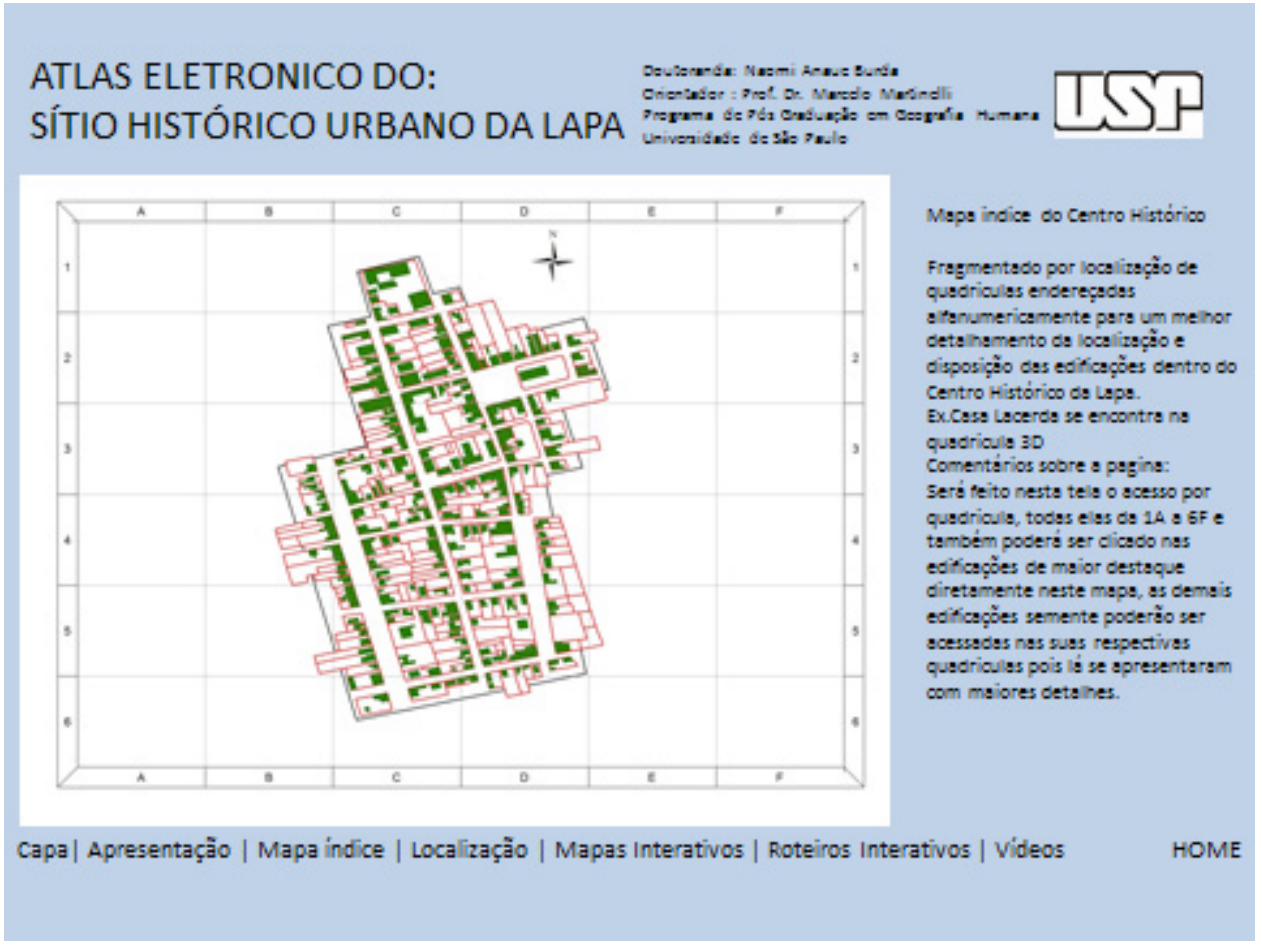

Figura 8. Esquema que divide as áreas do atlas protótipo para consulta Fonte: BURDA, 2012 


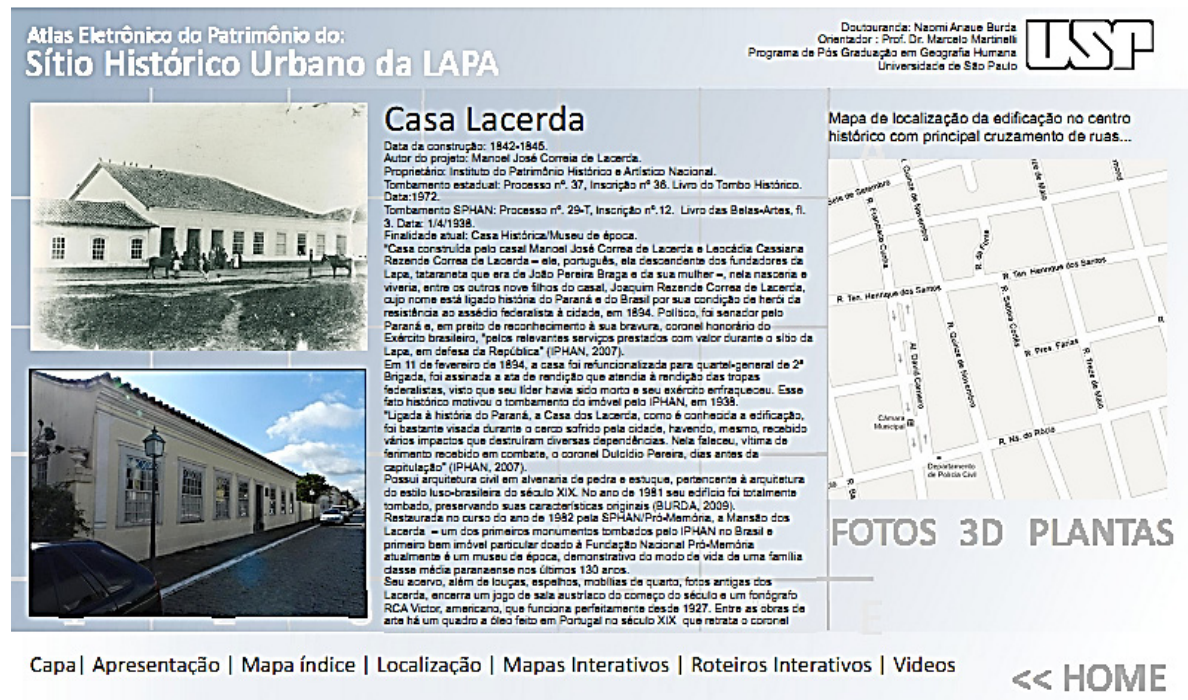

You created this PDF from an application that is not licansed to print to novaPDF printer (hitep:lifwww.novapdt.com)

Figura 9. Esquema que descreve características individuais dos casarões do atlas protótipo. Fonte: BURDA, 2012

\section{Considerações Finais}

Considerando que abordagens sobre patrimônio cultural na Geografia são em pouco número e mais raros ainda utilizando instrumentos de pesquisa da atualidade; tem-se como certa a validade de um estudo que servirá como referência teórica e metodológica para o conhecimento do patrimônio nesta área científica.

As contribuições da Geografia Humana para essa análise versarão sobre o papel da sociedade na questão de sua identificação com este patrimônio; a forma como é produzido e transformado esse espaço geográfico, o papel do geógrafo como agente de estudo desse espaço, como pode auxiliar na gestão patrimonial e outros.

As geotecnologias da atualidade, em casos como este, são pertinentes para a análise da gestão do patrimônio arquitetônico, pois permite ao pesquisador do tema uma modificação e atualização rápida dos conteúdos do atlas eletrônico e ao usuário é permitido um acesso facilitado e interativo do material. Os benefícios ao usuário estão presentes em conhecer a cidade antes de realizar uma visitação, ter um material que pode ser usado como metodologia para outras cidades patrimoniais e um recurso didático para a educação patrimonial.

A gestão de um espaço urbano, aqui enfatizando prioritariamente como uma área de patrimônio cultural, visa sugerir e encontrar soluções para uma melhor utilização, preservação, uso e divulgação do território de interesse, tanto para a população autóctone quanto para os turistas que visitam o local.

A definição de novos caminhos na construção do Atlas Eletrônico será elaborada durante o preparo da tese, mantendo a originalidade do projeto e a busca de novas 
rotas metodológicas a respeito de atlas eletrônicos.

A vantagem para o turista de pesquisar um local em um atlas eletrônico está na facilidade de acesso a um material prévio ao seu local de visitação. Pode-se pensar que este é um fator de repulsão do turista, pois vendo o local de visitação em casa o mesmo se desestimulará a realizar tal visita. Porém, tendo acesso a tais informações, esse instrumento poderá contribuir como fator de atração ao local, pois tendo um conhecimento dos atrativos e elementos do patrimônio cultural, por exemplo, o mesmo terá mais estímulo a procura-lo.
Com o acesso è internet fica facilitado a divulgação de mapas turísticos e também destacar os aspectos da cartografia, geotecnologias, turismo e patrimônio cultural. O diferencial é que o atlas na pretende ser apenas estático, mas tendo uma dinâmica que facilite o acesso ao leitor dos produtos.

Após testar e desenvolver uma metodologia para criação do atlas eletrônico, pretende-se que essa pesquisa seja consultada pelas pessoas que têm interesse de trabalhar com a gestão do território turístico e patrimonial através de instrumentos geográficos atuais.

\section{Referências}

ABADALA, N. A technique for building representation in oblique view maps of modern urban areas. The Cartographic Journal, v.46, n.2, p.104-114, 2009.

BERRY, J. K. Fundamental operations in computer-assisted map analysis. International Journal of Geographical Information Systems, v.1, n.2, p. 119-136, 1987.

BORGES, J. A.; FREITAS, M. I.; VENTORINI, S. E. Mapas táteis, dispositivos sonoros e aulas inclusivas com Mapavox. In: ALMEIDA, R. D. Novos rumos da cartografia escolar: currículo, linguagem e tecnologia. São Paulo: Contexto, 2011.

BURDA, N. A. Geotecnologias aplicadas à gestão do patrimônio cultural na cidade da Lapa (PR): construção do atlas digital do patrimônio arquitetônico. 2009.144 f. Dissertação (Mestrado em Gestão do Território) - Programa de Pós-Graduação em Geografia, Universidade Estadual de Ponta Grossa, Ponta Grossa, 2009.

BURROUGH, P. A. Principles of Geographical Information Systems for land resources assessment. 9. ed. London: Clarendon Press - Oxford, 1996.194p.

CÂMARA, G.; CASANOVA, M. A.; HEMERLY, A. S.; MAGALHÃES, G. C.; MEDEIROS, C. M. B. Anatomia de Sistemas de Informação geográfica. São José dos Campos: INPE, IBM Brasil, TELEBRÁS e UNICAMP, 1996. Disponível em <http://www. dpi.inpe.br/gilberto/livro/anatomia.pdf >. Acesso em: 10 ago. 2012.

CÂMARA, G.; DAVIS, C.; MONTEIRO, A. M. V. (Ed.). Introdução à Ciência da Geoinformação. São José dos Campos: INPE, 1999. Disponível em <http://www.dpi.inpe. br/gilberto/livro/introd/>. Acesso em: 10 ago. 2012. 
CARTER, G. B. Geographic Information Systems for geoscientists: modelling with GIS. Amsterdam: Elsevier Science, 1994.398p.

COPPOCK,J.T.; RHIND, D.W.The History of GIS.In:MAGUIRE D.J.; GOODCHILD M. F.; RHIND D. W. (Ed.). Geographical Information Systems: Principles and Applications, v.1, p.21-43, 1991.

DELAZARI, L. S. Modelagem e implementação de um atlas eletrônico interativo. 2004. 155 f. Tese (Engenharia)-Escola Politécnica. Universidade de São Paulo, USP, São Paulo, 2004.

FITZ, P. R. Geoprocessamento sem complicação. São Paulo: Oficina de Textos, 2008.160 p.

FORESMAN, T.W. The history of Geographic Information Systems, Prentice Hall, 1998.

GOODCHILD, M. F. Geographic information systems and cartography. Cartography, Santa Barbara: NCGIA, v. 19, n.1, p. 1-13, 1990.

GOODCHILD, M. F.; PROCTOR, J. D.; WRIGHT, D. J. Gis: Tool or Science? Desmystifying the persistent ambiguity of GIS as "Tool" versus "Science". Annals of the Association of American Geographers, v.87, n.2, p. 346-362, 1997.

HARVEY, D. Condição Pós Moderna: uma pesquisa sobre as origens da mudança cultural. São Paulo: Loyola, 1992.

LOUREIRO, M. D. Terá Marx algo a dizer sobre a informatização da sociedade? In: CONGRESSO NACIONAL DE INFORMÁTICA, 24., 1991, São Paulo. Anais...São Paulo: SUCESU.1991. p. 3-11.

MARTINELLI, M. As representações gráficas da geografia: os mapas temáticos. 1999. 218f. Tese (Livre Docência). Departamento de Geografia (FFLCH). São Paulo, 1999.

MARTINELLI, M. As cartografias e os atlas geográficos escolares. In: ENCONTRO NACIONAL DA ASSOCIAÇÃO DE PÓS-GRADUAÇÃO EM GEOGRAFIA, 9., 2011, Goiânia. [S.i]. Instituto de Estudos Sócioambientais, Universidade Federal de Goais, 2011.

MARTINELLI, M. Notas de aulas da disciplina de Representações Gráficas da Geografia: teoria e prática. Faculdade de Letras, Filosofia e Ciências Humanas, USP, 2011.

MARTIN, R.Teoria econômica e geografia humana.In: GREGORY,D.;MARTIN,R.; SMITH, G. (Org.). Geografia humana: sociedade, espaço e ciência social. Rio de Janeiro: Jorge Zahar, 1995.

MATIAS, L.F. Geotecnologias e patrimônio arquietônico: Potencialidades no mapeamento e análise para fins turísticos. In: LUCHIARI, M. T. D. P; OLIVEIRA, M. R. da S. (Org.). Geografia, Turismo e Patrimônio Cultural. São Paulo, 2010. p. 81-111. 
MATIAS, L. F. Por uma economia política das Geotecnologias. Revista Scripta Nova. Revista electrónica de Geografia y Ciencias Socialies, v.8, n.170 (52), p. 741-98, 2004.

MATIAS, L. F. Sistema de informações geográficas (SIG): teoria e método para representação do espaço geográfico. 2001. Tese (Doutorado em Geografia Humana)Departamento de Geociências (FFLCH). Universidade de São Paulo, São Paulo, 2001.

PINA, M. F.; SANTOS, S. M. Conceitos básicos de Sistemas de Informação Geográfica e Cartografia aplicados à saúde. Brasília: OPAS, 2000.

REGO, L. F. G. O uso de sistemas de informações geográficas para o estudo do espaço geográfico. In: RUA, J. (Org.). Paisagem, Espaço e Sustentabilidade. Rio de Janeiro: PUC, 2007. p. 83-90.

ROCHA, C.H. B. Geoprocessamento: tecnologia transdisciplinar.Juiz de Fora: UFJF, 2007.

RODRIGUES, M. Geoprocessamento. 1987. Tese (Livre Docência) - Escola Politécnica, Universidade de São Paulo, 1987.

SANTOS,M.A natureza do espaço: técnica e tempo, razão e emoção. São Paulo:USP,2009.384 p.

SANTOS, M.; SILVEIRA. O Brasil: território e sociedade no início do século XXI.12. ed. Rio de Janeiro: Record, 2008.

SMITH,T.R.; MENON, S.; STAR,J.L.; ESTES,J.E. Requirements and principles for the implementation and construction of large-scale geographic information systems. Journal of Geographical Information Systems, London: Taylor \& Francis, n.1, p.13-31, 1987.

TAYLOR, D. R. F. The art and science of cartography: the development of cartography and cartography for development. The Canadian Surveyor, Ottawa: The Canadian Institute of Surveying and Mapping, v. 41, v.3, 1987.

TAYLOR, D. R. F. Technology transfer and international development: some key issues for the mapping sciences. In: Simpósio Internacional sobre mapas e gráficos para deficientes visuais, 4., São Paulo: ACI/USP, 1994.

TEIXEIRA, A. L.A.; MORETTI, E.; CHRISTOFOLETTI, A. Introdução aos sistemas de informação geográfica. Rio Claro: Edição do Autor, 1992.79p.

TOMLIN, C. D. Geographic information systems and cartographic modeling. Englewood Cliffs: Prentice Hall, 1990. XVIII, 249 p.

VEREGIN, H. Computer innovation and adoption in geography. In: PICKLES, J. (Ed.). Ground truth the social implications of Geographic Information Systems. New York : The Guilford Press, 1995, 248p.

ZEILER, M. Modeling our World. Redlands: ESRI, 1999. 